\title{
A companion on the planet/brown dwarf mass boundary on a wide orbit discovered by gravitational microlensing
}

\author{
R. Poleski ${ }^{1,2}$, A. Udalski ${ }^{2}$, I. A. Bond ${ }^{3}$, J. P. Beaulieu ${ }^{4,5,6}$, C. Clanton ${ }^{7}$, S. Gaudi ${ }^{1}$, \\ M. K. Szymański ${ }^{2}$, I. Soszyński², P. Pietrukowicz ${ }^{2}$, Szymon Kozłowski ${ }^{2}$, J. Skowron ${ }^{2}$, Ł. Wyrzykowski ${ }^{2}$, \\ K. Ulaczyk ${ }^{8,2}$, D. P. Bennett ${ }^{9}$, T. Sumi ${ }^{10}$, D. Suzuki ${ }^{9}$, N. J. Rattenbury ${ }^{11}$, N. Koshimoto ${ }^{10}$, F. Abe ${ }^{12}$, Y. Asakura ${ }^{12}$,

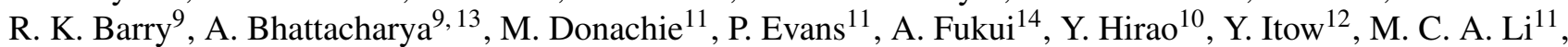 \\ C. H. Ling ${ }^{3}$, K. Masuda ${ }^{12}$, Y. Matsubara ${ }^{12}$, Y. Muraki ${ }^{12}$, M. Nagakane $^{10}$, K. Ohnishi ${ }^{15}$, C. Ranc $^{9}$, To. Saito ${ }^{16}$, \\ A. Sharan ${ }^{11}$, D. J. Sullivan ${ }^{17}$, P. J. Tristram ${ }^{18}$, T. Yamada ${ }^{10}$, T. Yamada ${ }^{19}$, A. Yonehara ${ }^{19}$, \\ V. Batista ${ }^{4}$, and J. B. Marquette ${ }^{4}$
}

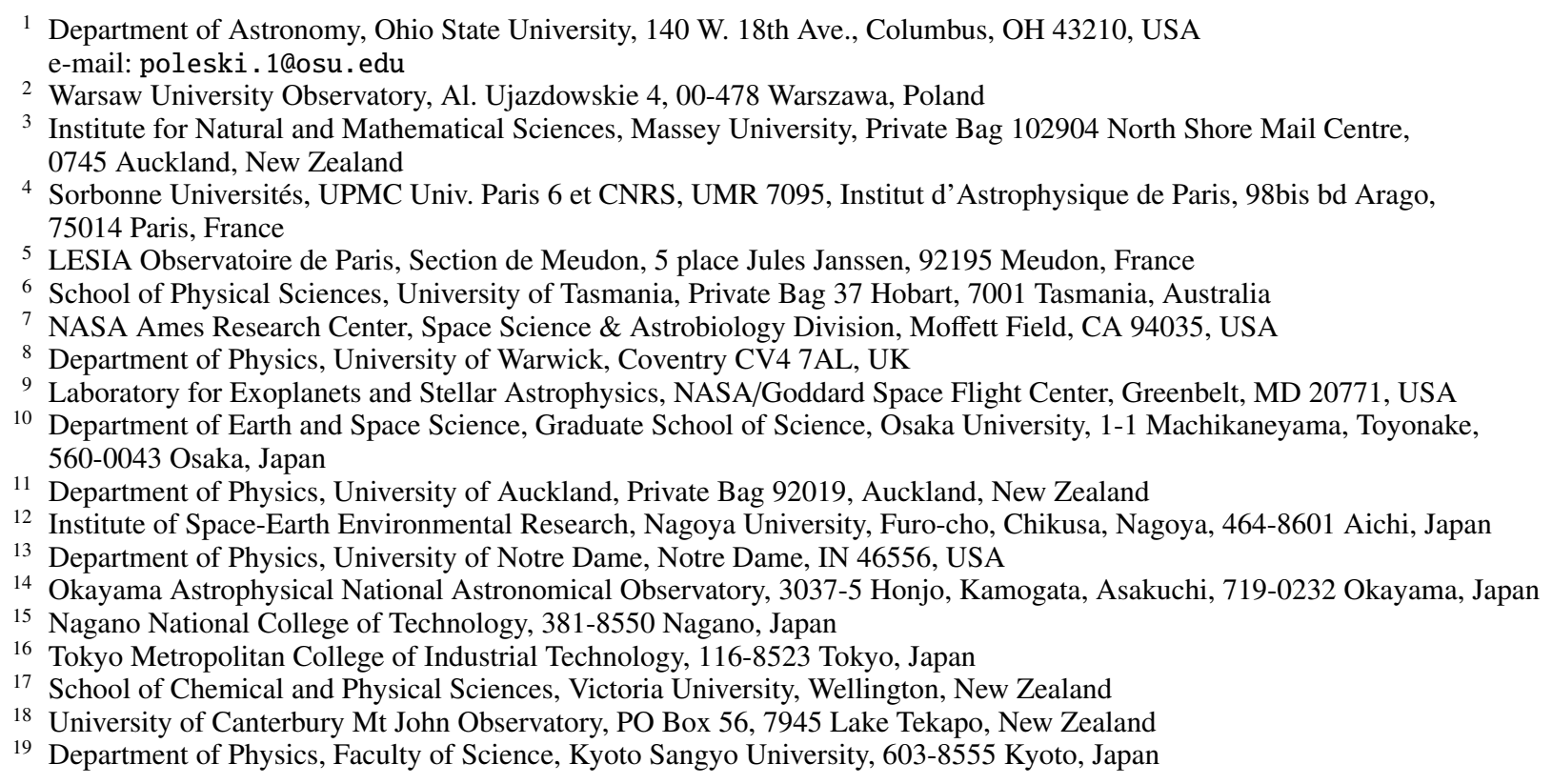

Received 3 April 2017 / Accepted 4 May 2017

\begin{abstract}
We present the discovery of a substellar companion to the primary host lens in the microlensing event MOA-2012-BLG-006. The companion-to-host mass ratio is 0.016 , corresponding to a companion mass of $\approx 8 M_{\mathrm{Jup}}\left(M_{*} / 0.5 M_{\odot}\right)$. Thus, the companion is either a high-mass giant planet or a low-mass brown dwarf, depending on the mass of the primary $M_{*}$. The companion signal was separated from the peak of the primary event by a time that was as much as four times longer than the event timescale. We therefore infer a relatively large projected separation of the companion from its host of $\approx 10 \mathrm{au}\left(M_{*} / 0.5 M_{\odot}\right)^{1 / 2}$ for a wide range $(3-7 \mathrm{kpc})$ of host star distances from the Earth. We also challenge a previous claim of a planetary companion to the lens star in microlensing event OGLE-2002-BLG-045.
\end{abstract}

Key words. gravitational lensing: micro - instrumentation: high angular resolution - planetary systems - brown dwarfs

\section{Introduction}

Brown dwarfs and planets are intrinsically faint objects and different detection techniques have to be used to explore a wide range of properties of these sub-stellar objects. Every detection technique has its own limitations and leads to a different kind of information when a new object is detected. Despite the large number of observational and theoretical studies (e.g., Beichman et al. 2014; Chauvin et al. 2015; Foreman-Mackey et al. 2016; Wilson et al. 2016), we are still far from a detailed understanding of the demographics of brown dwarf and planet populations that are also companions to stars. There is even a lack of consensus on the appropriate border line between planets and brown dwarfs (Boss et al. 2003; Grether \& Lineweaver 2006; Spiegel et al. 2011; Chabrier et al. 2014). The obvious way to increase our knowledge of sub-stellar mass objects is by discovering more objects and, in particular, by discovering and characterizing objects that question our current understanding of planet and brown dwarf formation and evolution. 
Here, we present the discovery of a binary system MOA2012-BLG-006L with a mass ratio of 0.016 and projected separation of roughly $10 \mathrm{au}$. Both components of the system were detected using the gravitational microlensing method. The advantage of this method is that it is sensitive to the mass of the objects, rather than their luminosity. As a result, microlensing enables the discovery of systems that are inaccessible to other techniques. First, the system distance of a few kpc prevents the detection of light from the lower-mass component via direct imaging. Second, the radial velocity signal of a long-period, lowmass companion to the faint host is out of reach of current techniques. Finally, the projected separation of about 10 au results in the extremely low probability of observing the transit, even if a population of similar systems was observed using photometric methods. We note that there are three other systems containing companions at planet/brown dwarf mass boundary that were discovered using microlensing: MOA-2007-BLG-197L (Ranc et al. 2015), MOA-2010-BLG-073L (Street et al. 2013), and MOA2011-BLG-322L (Shvartzvald et al. 2014). These three systems have smaller separations and higher mass ratios compared to the system reported here, MOA-2012-BLG-006L. The distribution of mass ratios for binary lens microlensing events was recently investigated by Shvartzvald et al. (2016). They found that the mass ratio distribution shows the minimum and this minimum is close to the mass ratio of MOA-2012-BLG-006L (0.016).

Explaining the formation of the MOA-2012-BLG-006L system poses significant challenges. The mass of the protoplanetary disc is typically $0.002-0.006$ of the host mass (Andrews et al. 2013), hence, any planet that forms in a protoplanetary disc that follows this observational trend cannot have a larger mass ratio. If the protoplanetary disc in MOA-2012-BLG-006L had a mass ratio close to the typical values, then the lower-mass object should be classified as a brown dwarf. However, there is a wide range of measured disc masses at fixed host mass (Andrews et al. 2013). In the extreme cases, estimated disc masses are close to 0.2 of the host mass (Andrews et al. 2009). In these extreme cases, the total mass of the disc is sufficient to form planets with mass ratios similar to MOA-2012-BLG-006Lb. The planetary formation scenario poses an additional question regarding how a planet so massive ended up on an orbit that is at least eight times larger than the snow line distance $(\approx 1.3 \mathrm{au}$ in this case). Most massive planets formed by core accretion should do so just beyond the snow line, where the protoplanetary disc is still relatively dense and ices can condense. Furthermore, if the planet formed via gravitational instability, we might expect it to be on an orbit wider by a factor of a few in semimajor axis (which, depending on the projection, it may actually be; Dodson-Robinson et al. 2009).

In the following section we describe photometric observations leading to the discovery of MOA-2012-BLG-006Lb. In Sect. 3 we analyze photometric data and derive the system properties using a Galactic model. The degeneracies in the microlensing model fitting are described in detail. The following section presents high-resolution observations of the event. Section 5 discusses another microlensing event (OGLE-2002-BLG-045) that showed a possible anomaly that could be fitted with a planetary model. We conclude that the anomaly was not real and there is no evidence for a planet. We end with conclusions.

\section{Photometric observations}

The microlensing event MOA-2012-BLG-006 was announced by the MOA group (Microlensing Observations in Astrophysics;
Bond et al. 2001) on Feb. 9, $2012\left(\mathrm{HJD}^{\prime} \equiv \mathrm{HJD}-2450000=\right.$ $5967.3)$ at $(\mathrm{RA}, \mathrm{Dec})=\left(18^{\mathrm{h}} 01^{\mathrm{m}} 46 \mathrm{~s} 1,-29^{\circ} 06^{\prime} 31^{\prime \prime} ! 6\right)$ (Galactic coordinates $l \approx 1: 64, b \approx-3.13)$. The event was found very early during the bulge observing season. During that time, bulge is visible only for a short time each night from any single site. The chances of discovering planets so early during the bulge observing season are low and most of the follow-up surveys do not start their normal operations before about a month later. Hence, survey observations are the only way to find planets that show their signatures so early in the season. The same event was alerted by the OGLE survey (Optical Gravitational Lensing Experiment; Udalski 2003) on Feb. 13, $2012\left(\mathrm{HJD}^{\prime}=5971\right)$ in the first batch of the microlensing events in 2012 and labeled OGLE-2012-BLG-0022. The OGLE and the MOA survey telescopes are well separated in geographic longitude, which allows coverage of different parts of the light curve. Below we describe the datasets produced by the two surveys.

The main photometric dataset comes from the OGLE survey, which uses a $1.3 \mathrm{~m}$ telescope located at Las Campanas Observatory (Chile). The telescope is equipped with the 32-CCD mosaic camera that gives a $1.4 \mathrm{deg}^{2}$ field of view (Udalski et al. 2015). There are eight photometric epochs during the anomaly (i.e., HJD' between 5960 and 5971) and 2306 more measurements during the 2012 bulge season - see light curve in Fig. 1. We also included 813 datapoints from 2011 in order to ensure that the baseline brightness is correctly measured. The OGLE survey performs most of the observations in the $I$-band and we use only these data for fitting. The $V$-band data do not cover the anomalous part of the light curve and are only used to derive source properties. The photometry was performed using the Difference Image Analysis (DIA) method (Alard 2000; Woźniak 2000). The photometric uncertainties were corrected using the prescription presented by Skowron et al. (2016). There are two relatively bright field stars that are very close to the event: 1.1 and 1.4 arcsec away with an $I$-band brightness of 17.6 and $16.2 \mathrm{mag}$, respectively. The two stars can affect the photometry of the event. Indeed, we found that seeing variations marginally influence brightness measurements - the target gets fainter by 0.005 mag for an increase in seeing FWHM of 1 arcsec. This effect was subtracted from the OGLE data.

The second dataset used comes from the MOA survey. The MOA survey operates a $1.8 \mathrm{~m}$ telescope situated at Mt. John Observatory (New Zealand). The filter used for observations is a custom wide-band optical filter. The camera consists of ten CCD detectors and gives a $2.2 \mathrm{deg}^{2}$ field of view (Sako et al. 2008). The MOA observing site has poorer weather and seeing conditions as compared to the OGLE site, but enables observations of microlensing events when Galactic bulge is invisible from Chile. Photometry was performed using the DIA method (Bond et al. 2001). MOA data for the analyzed event are more affected by the variable seeing and additionally show dependence of measured brightness on the airmass. Unfortunately, the specific way in which data are affected changes over time. We see these changes both during the event, and during the other observing seasons. In order not to include the affected data in the fit, we restricted the MOA dataset to the fourteen epochs that are closest to the anomaly, that is, from $\mathrm{HJD}^{\prime}=5961.1$ to 5969.2. Similar issues with a nearby star contaminating photometry of the microlensing event were faced by Gould et al. (2014) who analyzed the event OGLE-2013-BLG-0341. We note that the remaining MOA data will not improve accuracy of the fitted event properties, because with the exception of the anomaly, the event was of a low magnification and during bulge observing season the OGLE cadence of $20 \mathrm{~min}$ is more than sufficient to characterize the light curve. 


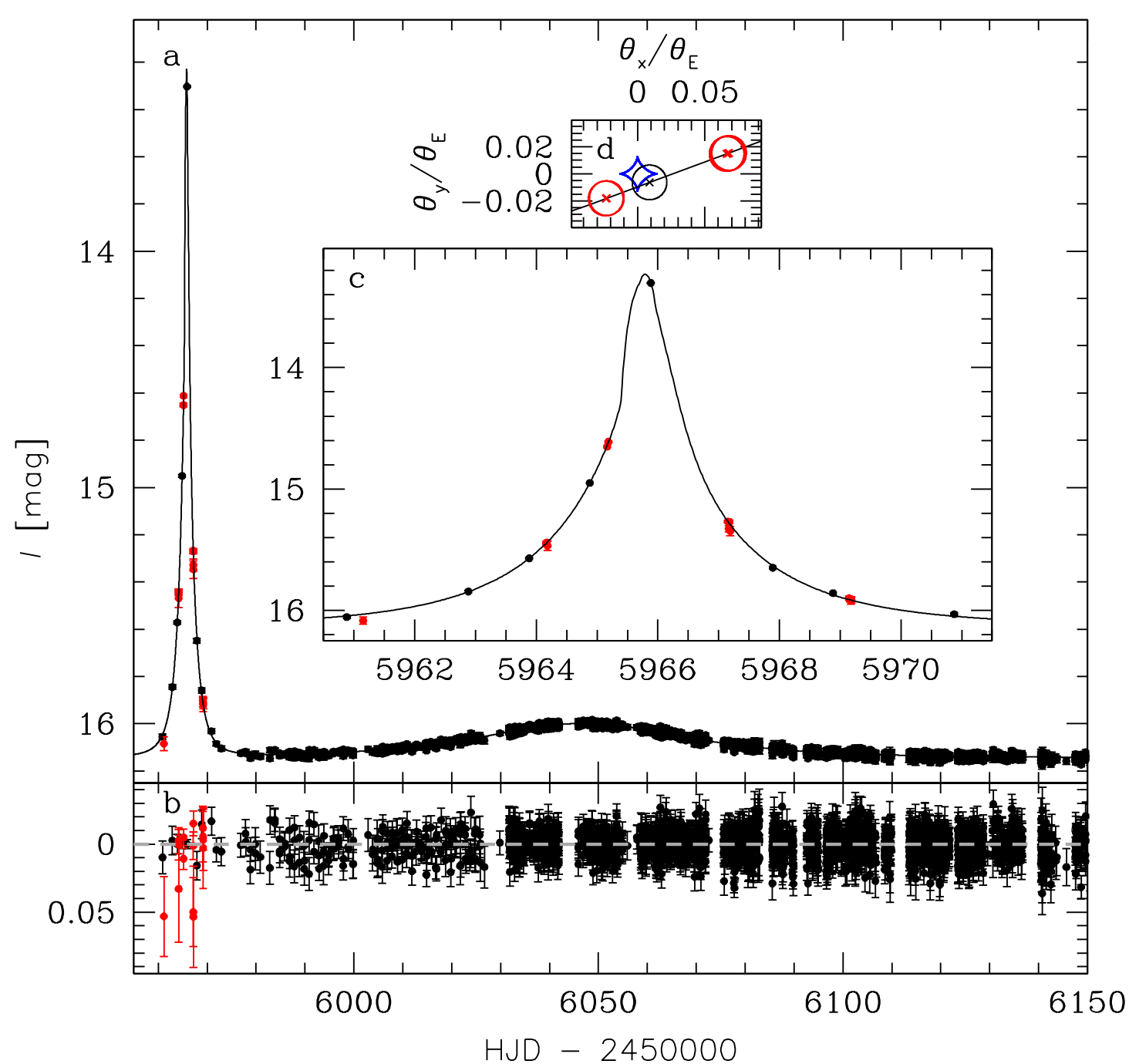

Fig. 1. Light curve of MOA-2012-BLG-006 = OGLE-2012-BLG-0022. OGLE and MOA data are marked using black and red points, respectively. Panel a) presents the 2012 light curve with both subevents. Panel b) shows the model residuals. Panel c) gives zoom-in on the anomaly. Panel d) presents the source trajectory (black line) relative to the planetary caustic (blue curve), which is at the origin of the coordinate system. The host star is located at $\left(\theta_{x} / \theta_{\mathrm{E}}, \theta_{y} / \theta_{\mathrm{E}}\right)=(4.17,0.0)$. Source positions from one OGLE night and two MOA nights are marked and are aligned with photometry shown in panel $\mathbf{c})$. The circles have a radius of $\rho$.

We also checked that the other bulge photometric survey operating at that time - VISTA Variables in the Vía Láctea (VVV; Minniti et al. 2010) - did not collect any data of this field during the anomaly.

\section{Analysis}

The light curve of MOA-2012-BLG-006 resembles a superposition of two point-source/point-lens microlensing events. Light curves of this type can be produced in two physically different scenarios (Gaudi 1998). First, the lens can be a single object and the source can be a binary system leading to two subevents with the same Einstein timescale $t_{\mathrm{E}}$. We tried to fit the binary source model to the observed light curve, but could not find a good fit.

After rejecting the binary source model, we are left with only one other possibility - the lens is a binary system and a single source is magnified (Gaudi 1998). If the two subevents are not significantly affected by the caustics (curves on which point source magnification is infinite), then the mass ratio of the two lens components is a square of the $t_{\mathrm{E}}$ ratio of the subevents. In the present case, a simple examination of the light curve by eye suggests that the lower mass object is either a planet or a brown dwarf if the host is a typical main sequence star. The first subevent has a higher magnification, even though it was caused by the lower mass object. The magnification of the subevent depends primarily on the impact parameter; not the lens mass.

To fit the microlensing model we evaluated magnification using the inverse ray shooting method for the highest magnified points and the hexadecapole approximation (Gould 2008; Pejcha \& Heyrovský 2009) for the adjacent parts of the light curve. We used the complex polynomial root solver by Skowron \& Gould (2012). Based on Claret \& Bloemen (2011) and source properties derived from the initially fitted model, we set the limb darkening coefficients to $\Gamma_{I}=0.502\left(u_{I}=0.602\right)$ and $\Gamma_{\mathrm{MOA}}=0.588\left(u_{\mathrm{MOA}}=0.681\right)$. We note that initial fitting was performed to the OGLE data only, but the results do not qualitatively differ from fits to the OGLE and MOA data.

We first tried to fit the model using Monte Carlo Markov chain (MCMC) that is typically used for the analysis of the microlensing events. We used MCMC implementation by Dong et al. (2007) and Poleski et al. (2014). Even though we run the MCMC multiple times with a number of settings, MCMC 
failed to produce a converging chain and hence we could not use it to fit a microlensing model. The triangle (or corner) plot showed that almost all two-parameter marginalized $\chi^{2}$ hypersurfaces had approximately ellipsoidal shapes but still the chain was not converging. The only exceptions were $\chi^{2}$ hypersurfaces where one of the parameters was the angular source radius relative to the Einstein ring radius $(\rho)$.

We fully understood the reason for failure in MCMC runs only after transforming the microlensing model to a different set of parameters. A default set of seven parameters that describes a binary lens model consists of the three point lens parameters, $\rho$, and the three parameters that describe the lens companion. The three point lens parameters are: $t_{0}$ - the epoch of minimum source-lens separation and; $u_{0}-$ the minimum separation relative to Einstein ring radius $\theta_{\mathrm{E}}$, and $t_{\mathrm{E}}$. The lens companion is described by: $\alpha$ - the angle between the lens axis and the source trajectory; $s$ - the separation of the lens components relative to $\theta_{\mathrm{E}}$; and $q-$ the mass ratio. In addition to the binary lens parameters that completely describe the magnification, the model also contains source and blending fluxes for each photometric system. The default binary lens parametrization is not optimal for fitting all the microlensing events because the parameters $\alpha, q$, and in many cases $s$ are not directly constrained by the light curves, in the sense that the observable properties of the microlensing event are not directly relatable to these parameters (Cassan 2008; Sumi et al. 2010; Skowron et al. 2011; Kains et al. 2012).

The event MOA-2012-BLG-006 shows two well-separated subevents and their parameters (maximum magnification, its epoch, and the length of the subevent) are well-constrained by the data, assuming the blending flux is known. Hence for fitting, we used, instead of the default set of parameters, $\rho$ and the three point lens parameters measured separately for each of the components $\left(t_{\mathrm{E}}\right)$ or relative to their caustics $\left(t_{0}\right.$ and $\left.u_{0}\right)$. We note that in this two-component parameterization either $u_{0,1}$ or $u_{0,2}$ has to be a signed quantity in order to make a distinction between the source passing both caustics on same or opposite sides (unlike in a point-source/point-lens model without parallax). The conversion between both binary lens parameterizations is based on simple geometry and the equation for distance between the central and the planetary caustics: $s-s^{-1}$ (Han 2006).

In the two-component parametrization, we can easily find and understand the very significant model degeneracies. We present the slice of $\chi^{2}$ hypersurface in Fig. 2. There are three local minima for different source trajectories and $\rho$ values. The best-fitting model has a $\rho$ of $\approx 0.012$ and the source trajectory that passes each caustic on a different side (see Fig. 1). The second best-fitting model has a much smaller source $(\rho \approx 0.001$ or even smaller) and the source passing through the center of the planetary caustic. The small source causes the characteristic U-shaped light curve (see the panel d of Fig. 2) but both highmagnification parts of the light curve (when the source crosses the caustic) that reach $I$-band magnitude of 12.3 are predicted to have occurred during the time when no data were taken. In this model, the brightest OGLE data point is taken close to the middle of the U-shaped trough. A Bayesian argument suggests that a model that predicts a large brightness variation during a time when no data were taken is a priori unlikely, although this argument alone cannot rule out such a model. However, we can exclude this model because it predicts unreasonably large relative lens-source geocentric proper motion $\mu=\theta_{\mathrm{E}} / t_{\mathrm{E}}=\theta_{\star} /\left(\rho t_{\mathrm{E}}\right)$

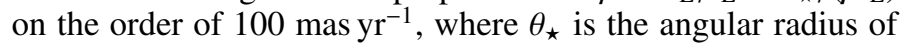
the source star equal to $5.68 \pm 0.34 \mu$ as (see Sect. 3.1). In the third solution, the source passes both caustics on the same side and has $\rho \approx 0.006$. We reject this solution because it is worse
Table 1. Double lens model parameters.

\begin{tabular}{llr}
\hline \hline Quantity & Unit & Value \\
\hline$t_{0}$ & & $6046.87 \pm 0.12$ \\
$u_{0}$ & & $1.432 \pm 0.032$ \\
$t_{\mathrm{E}}$ & $\mathrm{d}$ & $20.69 \pm 0.35$ \\
$\rho$ & & $0.0119_{-0.0023}^{+0.0016}$ \\
$\alpha$ & $\mathrm{deg}$ & $20.17 \pm 0.20$ \\
$s$ & & $4.405 \pm 0.069$ \\
$q$ & & $0.01650 \pm 0.00055$ \\
$F_{\text {s }} / F_{\text {base }}$ & & $0.981 \pm 0.054$ \\
\hline$\chi^{2} /$ d.o.f. & $2898.21 / 3130$ \\
\hline
\end{tabular}

Notes. The parameter $F_{\mathrm{s}} / F_{\text {base }}$ indicates ratio of source flux to baseline flux in the $I$-band. The value of $u_{0}$ is greater than one, hence, the host subevent would not normally be counted for the optical depth calculations.

than the first solution by $\Delta \chi^{2}=5.8\left(\Delta \chi^{2}=51.3\right.$ if MOA data are included).

Ultimately, we decided not to use the usual MCMC algorithm for fitting the model, but instead apply an alternative algorithm that is more suited to exploring degenerate multidimensional distributions - Multimodal Ellipsoidal Nested Sampling or MultiNest. The algorithm is described in detail and implemented by Feroz \& Hobson (2008) and Feroz et al. (2009, 2013). In brief, MultiNest approximates a volume of parameter space for which $\chi^{2}$ is below some limiting value $\chi_{0}^{2}$ by a set of $N$ points. We used $N=5000$ here for the final fitting, but reasonably good exploration of parameter space is achieved even for $N=500$ when considering only OGLE data. Both parameter estimation and model selection result from a single simulation in which $\chi_{0}^{2}$ is reduced from one step to the next. In every step, one of the $N$ points with the highest $\chi^{2}$ is replaced by a point that has lower $\chi^{2}$ and was found by trial-and-error. The trial-and-error procedure randomly samples a union of ellipsoids enclosing the $N$ points according to the prior (which was uniform in linear parameters).

For the final model fitting, we used both OGLE and MOA data and assumed $\rho>0.007$. The $\rho$ constraint is equivalent to

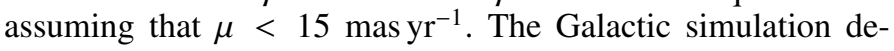
scribed below (with only $t_{\mathrm{E}}$ constrained) gives a probability of

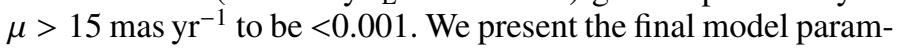
eters obtained using MultiNest in Table 1. Even though the fitting was performed in a two-component parametrization, all the parameters in default parametrization except $\rho$ show symmetric posterior distributions. We note that the event MOA-2012-BLG006 was used in a statistical analysis of the exoplanet mass ratio function by Suzuki et al. (2016). The parameters used there $\left(t_{\mathrm{E}}=21.13 \mathrm{~d}, u_{0}=1.3, q=0.01614, s=4.32\right)$ slightly differ from those found in the present work. The Suzuki et al. (2016) analysis was performed independently from the present analysis.

The problems with fitting the microlensing models described above are primarily caused by the poor coverage of the anomalous part of the light curve. Similar problems frequently appear during the analysis of poorly sampled anomalies. Jaroszynski \& Paczynski (2002) claimed that a single point anomaly in OGLE-2002-BLG-055 could be explained by the planetary model. Later, Gaudi \& Han (2004) showed that there is a plethora of models with non-planetary mass ratios that can fit the same light curve. Analysis of the planetary event MOA2007-BLG-192 revealed degenerate cusp approach and caustic crossing solutions that could not be efficiently sampled by 


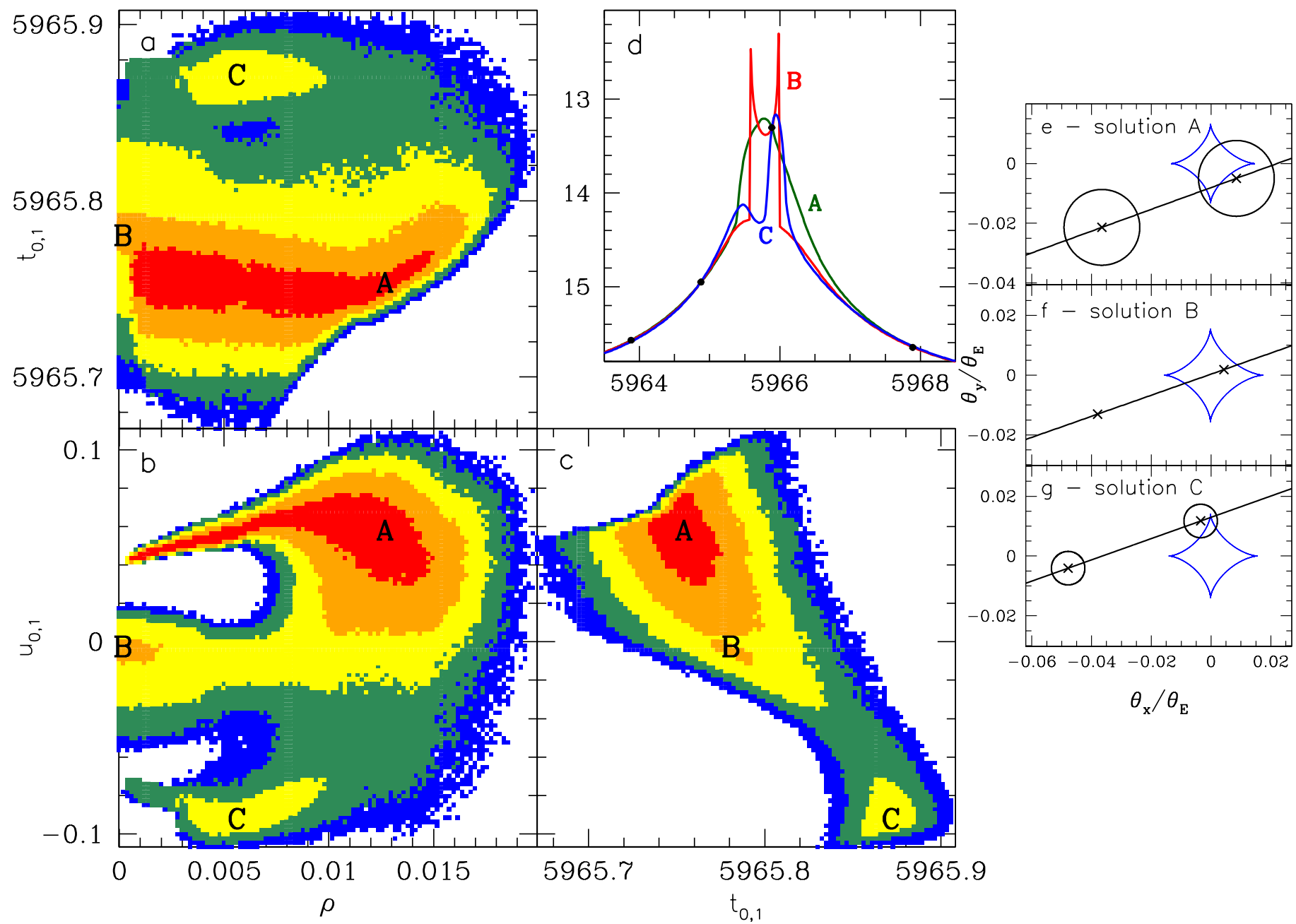

Fig. 2. Degenerate solutions for MOA-2012-BLG-006. Top center panel d) presents anomaly part of light curves for three modes A, B, and C. All three light curves predict the second subevent at $\mathrm{HJD}^{\prime}=6047$. Remaining large panels $\left.\left.\mathbf{a}\right)-\mathbf{c}\right)$ show the projection of the marginalized $\chi^{2}$ hypersurface. Red, orange, yellow, green, and blue points correspond to $\Delta \chi^{2}$ of $<1,<4,<9,<16$, and $<25$, respectively. Letters A, B, and C mark the three modes. We note that $u_{0,1}>0$ means that the companion and the host caustics are passed on opposite sides. The data presented on this plot are not the result of a single run, but a compilation of many simulations and were obtained using OGLE data only. Similar plot for OGLE and MOA data does not show significant differences except different levels of $\Delta \chi^{2}$. In particular, MOA data at HJD' $=5965.2$ and 5967.2 significantly contribute to preference of mode A over mode C. The small panels on the right $\mathbf{e})-\mathbf{g}$ ) show source trajectories of the three solutions relative to planetary caustic (blue curve). Crosses mark the source positions at epochs when OGLE data were taken. Circles have a radius of $\rho$. In the case of solution $\mathrm{B}$, the source size of $\rho=0.00016$ is too small to be seen.

MCMC runs (Bennett et al. 2008) because of the huge number of steps needed to cross the $\chi^{2}$ barrier between them. We predict that MultiNest can solve the remaining problems in analysis of these, and other, poorly sampled events.

Direct measurement of the lens mass, distance, and projected separation of the lens components requires microlensing parallax $\pi_{\mathrm{E}}$ (Gould 2000) to be measured. We cannot measure or even put meaningful constraints on $\pi_{\mathrm{E}}$ for MOA-2012-BLG-006 because the host subevent is too short and the value of $u_{0}$ is too large. The companion subevent could reveal the parallax signal only if it was sampled at a much higher cadence. Without a parallax measurement, we have to use source properties and Bayesian priors using a Galactic model to constrain the lens mass, distance, and projected separation of components.

\subsection{Source properties}

The lens mass and distance are crucial parameters for determining the nature of the lens system. These parameters cannot be directly derived from only the microlensing parameters like $t_{\mathrm{E}}$ and $\rho$. Microlensing events with finite source effects (and thus measured $\rho$ ) can only be used to estimate the physical properties of the lens if we can measure the angular Einstein ring radius $\theta_{\mathrm{E}}=\theta_{\star} / \rho$ and also measure the microlensing parallax or the lens flux. Here the estimate of $\theta_{\star}$ follows the method by Yoo et al. (2004). First, we construct the color-magnitude diagram for stars lying close to the event as presented in Fig. 3. Second, we measure the properties of the red clump (RC): $I_{\mathrm{RC}}=15.767 \pm 0.017 \mathrm{mag}$ and $(V-I)_{\mathrm{RC}}=2.024 \pm 0.007 \mathrm{mag}$. Third, by comparing these values with theoretical values, $I_{\mathrm{RC}, 0}=$ $14.381 \mathrm{mag}$ (found by interpolation of Table 2 from Nataf et al. 2013) and $(V-I)_{\mathrm{RC}, 0}=1.06 \mathrm{mag}$ (Bensby et al. 2011), we find extinction $A_{I}=1.386 \mathrm{mag}$ and reddening $E(V-I)=$ 0.964 mag. Fourth, we correct the source's unmagnified brightness $\left(I_{\mathrm{s}}=16.247 \mathrm{mag}\right.$ and $\left.V_{\mathrm{s}}=18.390 \mathrm{mag}\right)$ for extinction and obtain: $I_{\mathrm{s}, 0}=14.861 \mathrm{mag}$ and $V_{\mathrm{s}, 0}=16.040 \mathrm{mag}$. Fifth, the extinction-corrected brightness in visual bands is transformed to the near-infrared brightness of $K_{\mathrm{s}, 0}=13.314$ mag based on the intrinsic colors of giant stars (Bessell \& Brett 1988). Sixth, the 


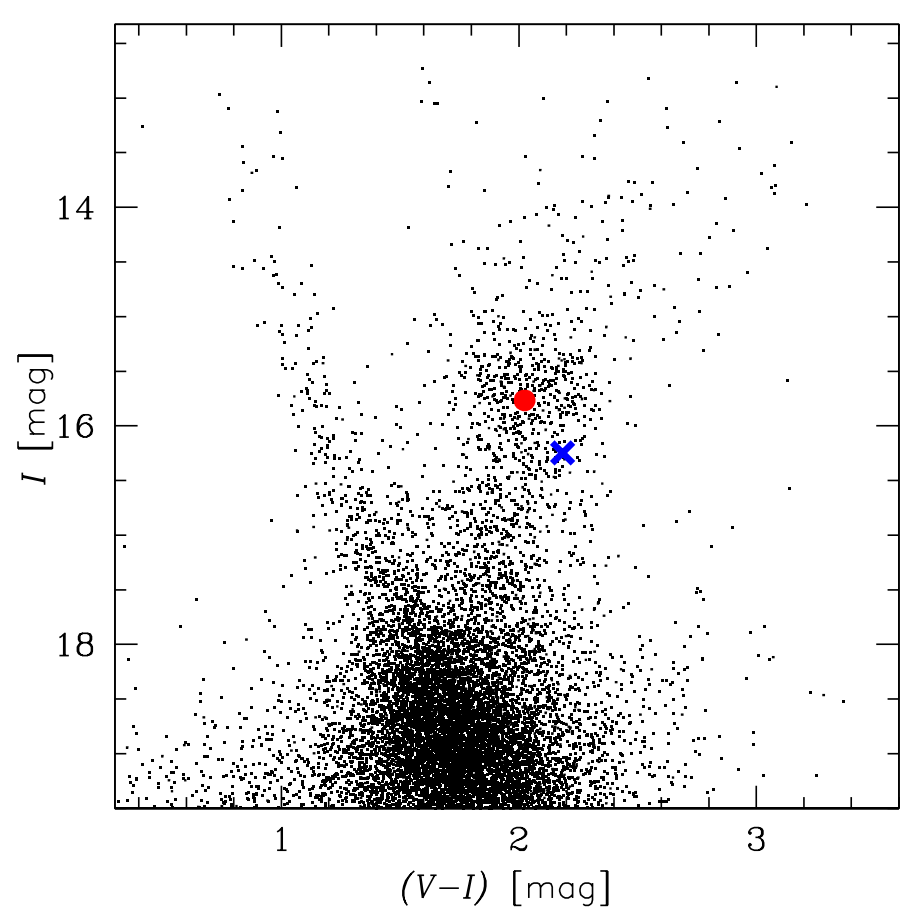

Fig. 3. Color-magnitude diagram for stars within $2^{\prime}$ around MOA-2012BLG-006. Red circle marks centroid of the red clump. The blue cross marks the position of the source.

angular source radius of $\theta_{\star}=5.68 \pm 0.36 \mu$ as is calculated using the relation between surface brightness and $(V-K)$ color by Kervella et al. (2004). This procedure results in $\theta_{\mathrm{E}}=\theta_{\star} / \rho=$ $0.489_{-0.038}^{+0.126}$ mas.

The event was also observed in $J$-band using adaptive optics (AO) at Keck telescope. Bessell \& Brett (1988) relations predict an extinction-free source brightness of $J_{\mathrm{s}, 0}=14.077 \pm$ $0.085 \mathrm{mag}$. The $J$-band extinction toward the event is $A_{J}=$ $0.58 \pm 0.16 \mathrm{mag}$ (Gonzalez et al. 2012). Hence, we predict observed source brightness of $J_{\mathrm{s}}=14.66 \pm 0.19 \mathrm{mag}$.

\subsection{Galactic model}

To derive the physical properties of the lens, we simulated microlensing events using the modified version of Galactic model by Clanton \& Gaudi (2014) and we refer the reader to that paper for a detailed description. The model includes lenses from Galactic disc with a double-exponential density profile and boxy Gaussian bulge. The line-of-sight projected velocity of the Earth is calculated for a peak of the anomaly. The lens mass distribution is the same as in Sumi et al. (2011) model 1 limited to the main sequence lenses: power laws with $\alpha=1.3$ for $0.08 \leq M / M_{\odot}<0.7$ and $\alpha=2.0$ for $0.7 \leq M / M_{\odot}<1.0$. Sources are placed at a distance of $7.8 \mathrm{kpc}$. The ensemble of simulated events is additionally weighted according to the measured $t_{\mathrm{E}}$ and $\theta_{\mathrm{E}}$. No constraint on the lens flux was applied. The resulting distributions are used to estimate the physical properties of the lens. We used the Astropy package (Astropy Collaboration et al. 2013) to analyze the simulation.

Figure 4 and Table 2 present the posterior distributions of the event parameters as derived from the Galactic model: $M_{\mathrm{h}}$ and $M_{\mathrm{c}}-$ mass of the host and companion, respectively, $a_{\perp}-$ projected separation of host and companion, $D_{1}-$ distance to the lens, and $\mu$-relative proper motion of lens and source. We note that $a_{\perp}=10.2$ au corresponds to deprojected semi-major axis
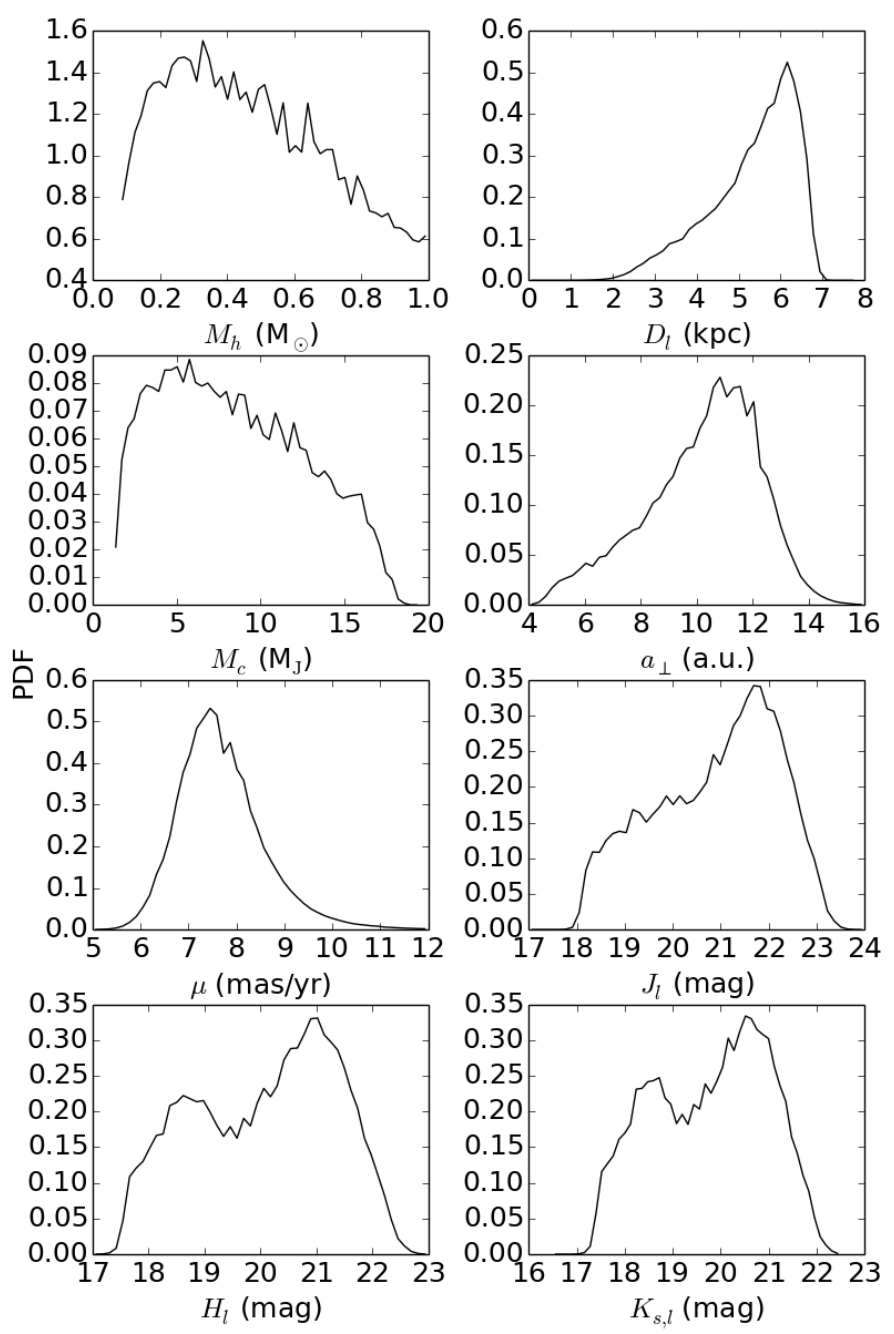

Fig. 4. Posterior distributions of parameters derived from the Galactic model. The three lower panels show predicted brightness of the lens in $J, H$, and $K_{\mathrm{s}}$ filters (2MASS system). The extinction of $A_{J}=0.58, A_{H}=$ 0.33 , and $A_{K_{\mathrm{s}}}=0.20$ (Gonzalez et al. 2012) were assumed independent of the lens distance.

Table 2. Posterior statistics for event parameters using a Bayesian prior and a Galactic model.

\begin{tabular}{llr}
\hline \hline Quantity & Unit & Value \\
\hline$M_{\mathrm{h}}$ & $M_{\odot}$ & $0.49_{-0.23}^{+0.27}$ \\
$M_{\mathrm{c}}$ & $M_{J}$ & $8.4_{-3.9}^{+4.6}$ \\
$D_{\mathrm{l}}$ & $\mathrm{kpc}$ & $5.3_{-1.3}^{+0.8}$ \\
$a_{\perp}$ & $\mathrm{au}$ & $10.2_{-2.4}^{+1.8}$ \\
$\mu$ & $\mathrm{mas} / \mathrm{yr}$ & $7.69_{-0.76}^{+1.1}$ \\
\hline
\end{tabular}

Notes. Mean values and $1-\sigma$ uncertainties are given.

(for a circular orbit with a random value of the cosine of the inclination) of $a=\sqrt{3 / 2} a_{\perp}=12.5 \mathrm{au}$. The probability that $M_{\mathrm{c}}$ is above the frequently assumed minimum brown dwarf mass of $13 M_{J}$ is 0.18 . Figure 4 also includes predictions of lens nearinfrared brightness based on Dotter et al. (2008) 6 Gyr isochrone for $[\mathrm{Fe} / \mathrm{H}]=0.0$ and $Y=0.27$. 


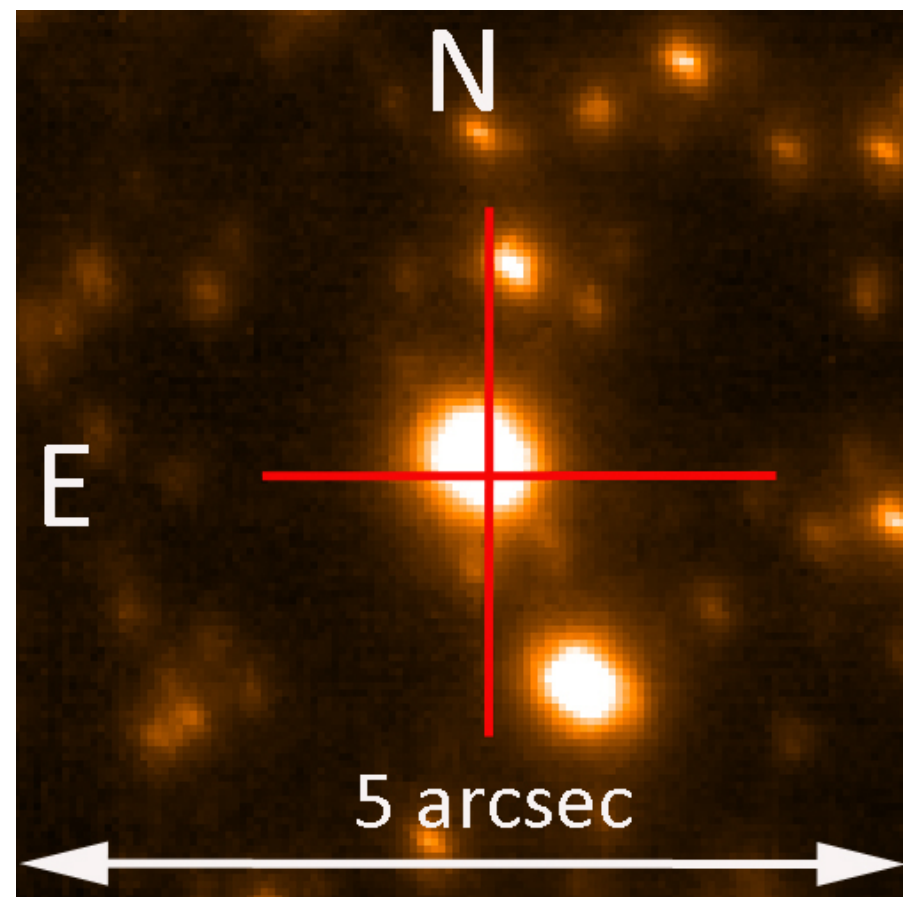

Fig. 5. Keck AO image of the event. The cross marks the expected position.

\section{High-resolution observations}

On July 18, 2013 (1.2 yr after peak of the event) we observed the microlensing event MOA-2012-BLG-006 with Near Infrared Camera 2 (NIRC-2) AO system mounted on the Keck-II telescope. We used the wide field $\left(40^{\prime \prime} \times 40^{\prime \prime}\right)$ camera with a pixel scale of 0.04 arcsec and $J$-band filter. We took four frames with an exposure time of $3 \times 10 \mathrm{~s}$ at each of the five dithered positions. We corrected for dark and flat fields using standard procedure and stacked the images using SWarp program from the AstrOmatic suite of astronomy tools (Bertin 2010). The full width at half maximum was 0.2 arcsec. The aperture photometry was performed by running SExtractor (Bertin \& Arnouts 1996) software. The photometric and astrometric calibration of the Keck images requires additional data and for this purpose we used VVV data. The VVV survey observed in $J, H$, and $K$ bands at the $4 \mathrm{~m}$ VISTA telescope at Paranal Observatory (Chile). To process VVV images we followed the procedure described by Beaulieu et al. (2016), which includes calibration of photometry and astrometry to 2MASS system (Skrutskie et al. 2006).

The source star is clearly identified on Keck image at the expected position (see Fig. 5). We note that there is no significant blend at the sub-arcsec separation. The VVV brightness is $J_{V V V}=14.72 \pm 0.02, H_{V V V}=13.88 \pm 0.02$, and $K_{V V V}=$ $13.62 \pm 0.03$. Based on cross-identification of the same stars in Keck and VVV data we estimate the error in absolute calibration of Keck photometry of $0.015 \mathrm{mag}$. The brightness measured on Keck image and calibrated to VVV data is $J_{\text {Keck }}=14.70 \pm 0.03$.

We can compare the total object brightness measured from the Keck image with the lens and source brightness estimated before. The fiducial lens mass from our Galactic model $\left(0.49 M_{\odot}\right)$ corresponds to absolute brightness on main sequence of $J_{l, 0}=$ 6.20 (Dotter et al. 2008). The fiducial lens distance is $5.3 \mathrm{kpc}$, hence, it should be behind almost all extinction observed in this field. The expected brightness of the lens is hence $J_{1}=20.40$ (see also Fig. 4). The optical data and VVV extinction predict the source brightness of $J_{\mathrm{s}}=14.66 \pm 0.19$. Hence, the lens is on the order of 6 mag fainter than the source and its contribution to the total light $(0.004 \mathrm{mag})$ is much smaller than the uncertainty in $J_{\mathrm{s}}$. The object brightness measured on the Keck image $J_{\text {Keck }}=14.70 \pm 0.03$ is marginally brighter than the predicted source brightness $J_{\mathrm{s}}=14.66 \pm 0.19$, that is, no light from the lens is detected. However, there is no statistical difference between the two measurements, primarily due to the large uncertainty of $J$-band extinction.

\section{Solving the mystery of OGLE-2002-BLG-045}

Skowron et al. (2009) analyzed a number of microlensing events that were observed to repeat, that is, show more than one brightening episode. The second episode can be caused by either a companion to the lens or a companion to the source. The interesting finding by Skowron et al. (2009) was that the event OGLE2002-BLG-045 showed two consecutive OGLE datapoints that are well separated from the main event and significantly brighter than the baseline. The two observations happened only four days apart $\left(\mathrm{HJD}^{\prime}=2455.7\right.$ and 2459.6) and were separated a few days from the previous $\left(\mathrm{HJD}^{\prime}=2448.7\right)$ and the following $\left(\mathrm{HJD}^{\prime}=2463.6\right)$ observations, which both were at the baseline. The short time between the two anomaly observations compared to $t_{\mathrm{E}}=26.4 \mathrm{~d}$ suggests that the anomaly could have been caused by a planetary companion to the lens $(q=0.008$ and $s=3.958$ ). As was pointed out by Skowron et al. (2009), the only evidence for existence of the planet were the two data points brighter than the baseline, and therefore the planet detection of the planetary companion was questionable. Because of this ambiguity, the putative planet OGLE-2002-BLG-045Lb is not normally considered on the lists of the known microlensing planets (e.g., Zhu et al. 2014; Penny et al. 2016; Mróz et al. 2017).

In order to verify the planetary signal in OGLE-2002BLG-045 we performed photometry of the archival data acquired by the previous phase of the MOA survey (MOA-I; Yanagisawa et al. 2000; Bond et al. 2001). No signal of the planet was found. We also visually verified the OGLE images that resulted in two anomalous points and found that they were taken in non-photometric conditions. Skowron et al. (2009) inspected 4120 events, hence it is not surprising that in this sample they found an event with two consecutive erroneous measurements separated by a few $t_{\mathrm{E}}$ from the event peak. We conclude that there is no convincing evidence that OGLE-2002BLG-045L has a wide separation planet and the two data points brighter than baseline are simply observational artifacts.

\section{Conclusions}

We present the discovery of MOA-2012-BLG-006Lb - an object a few times more massive than Jupiter, which can be classified based on its mass either as a planet (most probable scenario) or a brown dwarf (if its mass is at the high end of the derived distribution). We detected microlensing signal not only due to this object but also due to its host star. The mass ratio is above 0.01 , that is, higher than the typical mass ratio of a protoplanetary disc to the parent star. Hence, the lower-mass object could have formed independently and thus resembles brown dwarfs, even if its mass is smaller than the commonly assumed boundary of $13 M_{J}$.

The lack of parallax constraint precludes a direct measurement of the lens mass and projected separation of its components, but the large ratio between the two subevents' time separation and the event timescale suggests the companion is on a very wide orbit. Bayesian inference using a Galactic model results in 
a $0.49 \pm 0.25 M_{\odot}$ host orbited by a $8.4 \pm 4.3 M_{J}$ companion at a projected separation of $10.2 \pm 2.2$ au. The projected separation relative to Einstein ring radius of $s=4.4$ is the second largest among low-mass companions found by the microlensing technique after OGLE-2008-BLG-092 ( $s=5.3$; Poleski et al. 2014) and preceding MOA-2007-BLG-400 ( $s=2.9$ if wider of the two solutions is true; Dong et al. 2009).

The properties of MOA-2012-BLG-006Lb are similar to the small number of objects, either high-mass planets or brown dwarfs, that have been discovered around $M$ stars via direct imaging and which typically have orbital separations of tens to hundreds of au (see e.g., Table 1 of Lannier et al. 2016). The masses of such planets tend to be at least the same order of magnitude as the total amount of mass that comprised the protoplanetary disk within (and from) which we would expect them to have formed, presenting a challenge to our current understanding of giant planet formation. Nevertheless, the discovery of MOA-2012-BLG-006Lb suggests that whatever mechanisms are responsible for the formation of such objects, they seem to operate similarly in the immediate Solar neighborhood (where direct imaging finds them) and in other parts of the Galaxy, several kpc away (where only microlensing is sensitive to their detection).

The AO image of the event was taken using the Keck NIRC-2 camera. The contribution of the lens flux to the total observed flux could not be measured due to large uncertainty in extinction and the fact that the lens is expected to be substantially fainter than the source.

We also showed that the anomaly observed in another event OGLE-2002-BLG-045 - is of instrumental origin. Hence, there is no planetary signature in that event.

Acknowledgements. The authors would like to thank Prof. A. Gould for consultation. The OGLE project has received funding from the National Science Centre, Poland, grant MAESTRO 2014/14/A/ST9/00121 to A.U. OGLE Team acknowledges Profs. M. Kubiak and G. Pietrzyński, former members of the team, for their contribution to the collection of the OGLE photometric data over the past years. J.P.B. and J.B.M. gratefully acknowledge support from ESO's DGDF 2014. C.C. was supported by an appointment to the NASA Postdoctoral Program at NASA Ames Research Center, administered by Universities Space Research Association under contract with NASA.

\section{References}

Alard, C. 2000, A\&AS, 144, 363

Andrews, S. M., Wilner, D. J., Hughes, A. M., Qi, C., \& Dullemond, C. P. 2009, ApJ, 700, 1502

Andrews, S. M., Rosenfeld, K. A., Kraus, A. L., \& Wilner, D. J. 2013, ApJ, 771, 129

Astropy Collaboration, Robitaille, T. P., Tollerud, E. J., et al. 2013, A\&A, 558, A33

Beaulieu, J.-P., Bennett, D. P., Batista, V., et al. 2016, ApJ, 824, 83

Beichman, C., Gelino, C. R., Kirkpatrick, J. D., et al. 2014, ApJ, 783, 68

Bennett, D. P., Bond, I. A., Udalski, A., et al. 2008, ApJ, 684, 663

Bensby, T., Adén, D., Meléndez, J., et al. 2011, A\&A, 533, A134

Bertin, E. 2010, SWarp: Resampling and Co-adding FITS Images Together, Astrophysics Source Code Library [ascl .net/1010.068]
Bertin, E., \& Arnouts, S. 1996, A\&AS, 117, 393

Bessell, M. S., \& Brett, J. M. 1988, PASP, 100, 1134

Bond, I. A., Abe, F., Dodd, R. J., et al. 2001, MNRAS, 327, 868

Boss, A. P., Basri, G., Kumar, S. S., et al. 2003, Brown Dwarfs, IAU Symp., 211, 529

Cassan, A. 2008, A\&A, 491, 587

Chabrier, G., Johansen, A., Janson, M., \& Rafikov, R. 2014, Protostars and Planets VI (Tucson: University of Arizona Press), 619

Chauvin, G., Vigan, A., Bonnefoy, M., et al. 2015, A\&A, 573, A127

Clanton, C., \& Gaudi, B. S. 2014, ApJ, 791, 90

Claret, A., \& Bloemen, S. 2011, A\&A, 529, A75

Dodson-Robinson, S. E., Veras, D., Ford, E. B., \& Beichman, C. A. 2009, ApJ, 707, 79

Dong, S., Udalski, A., Gould, A., et al. 2007, ApJ, 664, 862

Dong, S., Bond, I. A., Gould, A., et al. 2009, ApJ, 698, 1826

Dotter, A., Chaboyer, B., Jevremović, D., et al. 2008, ApJS, 178, 89

Feroz, F., \& Hobson, M. P. 2008, MNRAS, 384, 449

Feroz, F., Hobson, M. P., \& Bridges, M. 2009, MNRAS, 398, 1601

Feroz, F., Hobson, M. P., Cameron, E., \& Pettitt, A. N. 2013, ArXiv e-prints [arXiv: 1306.2144]

Foreman-Mackey, D., Morton, T. D., Hogg, D. W., Agol, E., \& Schölkopf, B. 2016, AJ, 152, 206

Gaudi, B. S. 1998, ApJ, 506, 533

Gaudi, B. S., \& Han, C. 2004, ApJ, 611, 528

Gonzalez, O. A., Rejkuba, M., Zoccali, M., et al. 2012, A\&A, 543, A13

Gould, A. 2000, ApJ, 542, 785

Gould, A. 2008, ApJ, 681, 1593

Gould, A., Udalski, A., Shin, I.-G., et al. 2014, Science, 345, 46

Grether, D., \& Lineweaver, C. H. 2006, ApJ, 640, 1051

Han, C. 2006, ApJ, 638, 1080

Jaroszynski, M., \& Paczynski, B. 2002, Acta Astron., 52, 361

Kains, N., Browne, P., Horne, K., Hundertmark, M., \& Cassan, A. 2012, MNRAS, 426, 2228

Kervella, P., Bersier, D., Mourard, D., et al. 2004, A\&A, 428, 587

Lannier, J., Delorme, P., Lagrange, A. M., et al. 2016, A\&A, 596, A83

Minniti, D., Lucas, P. W., Emerson, J. P., et al. 2010, New Astron., 15, 433

Mróz, P., Han, C., Udalski, A., et al. 2017, AJ, 153, 143

Nataf, D. M., Gould, A., Fouqué, P., et al. 2013, ApJ, 769, 88

Pejcha, O., \& Heyrovský, D. 2009, ApJ, 690, 1772

Penny, M. T., Henderson, C. B., \& Clanton, C. 2016, ApJ, 830, 150

Poleski, R., Skowron, J., Udalski, A., et al. 2014, ApJ, 795, 42

Ranc, C., Cassan, A., Albrow, M. D., et al. 2015, A\&A, 580, A125

Sako, T., Sekiguchi, T., Sasaki, M., et al. 2008, Exp. Astron., 22, 5

Shvartzvald, Y., Maoz, D., Kaspi, S., et al. 2014, MNRAS, 439, 604

Shvartzvald, Y., Maoz, D., Udalski, A., et al. 2016, MNRAS, 457, 4089

Skowron, J., \& Gould, A. 2012, ArXiv e-prints [arXiv: 1203.1034]

Skowron, J., Wyrzykowski, Ł., Mao, S., \& Jaroszyński, M. 2009, MNRAS, 393, 999

Skowron, J., Udalski, A., Gould, A., et al. 2011, ApJ, 738, 87

Skowron, J., Udalski, A., Kozłowski, S., et al. 2016, Acta Astron., 66, 1

Skrutskie, M. F., Cutri, R. M., Stiening, R., et al. 2006, AJ, 131, 1163

Spiegel, D. S., Burrows, A., \& Milsom, J. A. 2011, ApJ, 727, 57

Street, R. A., Choi, J.-Y., Tsapras, Y., et al. 2013, ApJ, 763, 67

Sumi, T., Bennett, D. P., Bond, I. A., et al. 2010, ApJ, 710, 1641

Sumi, T., Kamiya, K., Bennett, D. P., et al. 2011, Nature, 473, 349

Suzuki, D., Bennett, D. P., Sumi, T., et al. 2016, ApJ, 833, 145

Udalski, A. 2003, Acta Astron., 53, 291

Udalski, A., Szymański, M. K., \& Szymański, G. 2015, Acta Astron., 65, 1

Wilson, P. A., Hébrard, G., Santos, N. C., et al. 2016, A\&A, 588, A144

Woźniak, P. R. 2000, Acta Astron., 50, 421

Yanagisawa, T., Muraki, Y., Matsubara, Y., et al. 2000, Exp. Astron., 10, 519

Yoo, J., DePoy, D. L., Gal-Yam, A., et al. 2004, ApJ, 603, 139

Zhu, W., Penny, M., Mao, S., Gould, A., \& Gendron, R. 2014, ApJ, 788, 73 